\title{
Face-induced expectancies influence neural mechanisms of performance monitoring
}

\author{
Roman Osinsky $^{1} \cdot$ Jennifer Seeger $^{1} \cdot$ Patrick Mussel $^{1} \cdot$ Johannes Hewig $^{1}$
}

Published online: 2 November 2015

(C) Psychonomic Society, Inc. 2015

\begin{abstract}
In many daily situations, the consequences of our actions are predicted by cues that are often social in nature. For instance, seeing the face of an evaluator (e.g., a supervisor at work) may activate certain evaluative expectancies, depending on the history of prior encounters with that particular person. We investigated how such face-induced expectancies influence neurocognitive functions of performance monitoring. We recorded an electroencephalogram while participants completed a time-estimation task, during which they received performance feedback from a strict and a lenient evaluator. During each trial, participants first saw the evaluator's face before performing the task and, finally, receiving feedback. Therefore, faces could be used as predictive cues for the upcoming evaluation. We analyzed electrocortical signatures of performance monitoring at the stages of cue processing, task performance, and feedback reception. Our results indicate that, at the cue stage, seeing the strict evaluator's face results in an anticipatory preparation of fronto-medial monitoring mechanisms, as reflected by a sustained negative-going amplitude shift (i.e., the contingent negative variation). At the performance stage, face-induced expectancies of a strict evaluation rule led to increases of early performance monitoring signals (i.e., frontal-midline theta power). At the final stage of feedback reception, violations of outcome expectancies differentially affected the feedback-related negativity and frontalmidline theta power, pointing to a functional dissociation between these signatures. Altogether, our results indicate that evaluative expectancies induced by face-cues lead to
\end{abstract}

Roman Osinsky

roman.osinsky@uni-wuerzburg.de

1 Department of Psychology, Julius-Maximilians-University, Marcusstr. 9-11, 97070 Würzburg, Germany adjustments of internal performance monitoring mechanisms at various stages of task processing.

Keywords Posterior medial frontal cortex · Frontal-midline theta $\cdot$ Error-related negativity $\cdot$ Feedback-related negativity . Contingent negative variation

\section{Introduction}

Continuous monitoring of our actions and their consequences is a crucial function of the brain, enabling fast and efficient cognitive and behavioral adaptations under changing environmental conditions. Previous research has repeatedly demonstrated that neural mechanisms involved in such performance monitoring functions are subject to action-outcome expectancies. In particular, numerous recent studies have shown that electrocortical correlates of performance monitoring in the scalp-recorded electroencephalogram (EEG) are sensitive to the predictive value of cue stimuli preceding action outcomes (e.g., Bellebaum \& Daum, 2008; Chase, Swainson, Durham, Benham, \& Cools, 2011; Hauser et al., 2014; Hewig et al., 2007; Martin \& Potts, 2011; Mas-Herrero \& Marco-Pallarés, 2014; Pfabigan, Alexopoulos, Bauer, \& Sailer, 2011; Pfabigan et al., 2015; Potts, Martin, Burton, \& Montague, 2006; Walsh \& Anderson, 2011; Wu \& Zhou, 2009; Xu et al., 2011). In the majority of these studies, the indicative cues were simple visual stimuli like, for instance, geometrical shapes, letters, or colors. In daily life, however, the outcomes of our actions are rather predicted by more complex stimuli that are often social in nature. As our behavior is frequently assessed by other human beings (e.g., family members, supervisors at work, lecturers at university), the faces of these evaluators may serve as visual cues, informing us about the probability of positive or negative action outcomes in a given situation. For instance, 
an evaluator's face may generally activate certain expectations when this person is known for either a lenient evaluative style (high probability of positive action outcome) or more strict evaluative style (high probability of negative action outcomes). Although it seems likely that the effects of such face cues on performance monitoring are very similar to those of more simple visual cues, this is still an issue that needs to be addressed empirically. In the present study, we therefore investigated whether and how face-induced and reputationbased expectancies influence electrocortical correlates of performance monitoring during a prototypical situation of actionassessment. Moreover, while most prior studies have focused on single stages of task processing, the used task design in our study allowed us to analyze performance monitoring functions at different stages, that is, the cue stage, the performance stage, and the feedback stage.

\section{EEG signatures of performance monitoring and their relation to outcome expectancies}

Before we outline our research question in more detail, it seems reasonable to briefly summarize previous relevant findings on electrocortical correlates of performance monitoring in the EEG and their relation to outcome expectancies. In tasks where the correctness of behavior can be directly inferred from behavior itself, a midfrontal negative-going potential occurs about 50 to $100 \mathrm{~ms}$ following errors and is therefore commonly referred to as error-related negativity (ERN; Falkenstein, Hohnsbein, Hoormann, \& Blanke, 1991; Gehring, Goss, Coles, Meyer, \& Donchin, 1993). A similar deflection has been observed about 200 to $300 \mathrm{~ms}$ following the onset of a feedback stimulus that signals a suboptimal performance or unfavorable action outcome, that is, the feedback-related negativity (FRN; Gehring \& Willoughby, 2002; Miltner, Braun, \& Coles, 1997). As has been shown by electrical source analyses and combined EEG-fMRI measurement, both the ERN and FRN are probably generated in the posterior medial frontal cortex (pMFC; e.g., Debener et al., 2005; Gehring \& Willoughby, 2002; Hauser et al., 2014; Miltner et al., 1997), which is a key region of the neural performance monitoring system, integrating manifold information and signaling the need for adaptive control (Shackman et al., 2011; Ullsperger, Danielmeier, \& Jocham, 2014). More recently, it has been proposed that the ERN and FRN are manifestations of oscillatory theta $(4-8 \mathrm{~Hz})$ activity. Accordingly, behavioral errors and unfavorable feedbacks induce increased fronto-medial theta (FM $\theta$ ) EEG power in the time range of the ERN and FRN, respectively (e.g., Cavanagh, Zambrano-Vazquez, \& Allen, 2012; Cohen, Elger, \& Ranganath, 2007; Luu, Tucker, \& Makeig, 2004; Mussel, Reiter, Osinsky, \& Hewig, 2015; Trujillo \& Allen, 2007). This FM $\theta$ activity may act as a generic "need for control" signal of the pMFC, instantiating control adjustments in adjacent and more distal regions like the lateral prefrontal cortex, sensory cortices, or motor regions (Cavanagh \& Frank, 2014).

According to a prominent theory, both the ERN and FRN reflect negative reward-prediction error (RPE) signals (i.e., an outcome is worse than expected), which are used by the pMFC for adaptations in action selection (Holroyd \& Coles, 2002). This reinforcement-learning theory has stimulated a wealth of research, with most studies focusing on the outcome-locked FRN and its link to action-outcome expectancies (for overviews, see, Sambrook \& Goslin, 2015; Ullsperger et al., 2014; Walsh \& Anderson, 2012). In several of these works, outcome expectancies were experimentally induced in a trial-by-trial manner by simple visual cue stimuli preceding the outcomes. Some of these studies indicate that FRN amplitude varies with the degree of signed RPEs (i.e., more negative FRN amplitude when the outcome is worse than expected and/or more positive FRN amplitude when the outcome is better than expected; e.g., Bellebaum \& Daum, 2008; Chase et al., 2011; Hewig et al., 2007; Martin \& Potts, 2011; Xu et al., 2011). However, other studies rather point to an association between FRN and unsigned RPEs (i.e., generally more negative FRN when the outcome is other than expected; e.g., Hauser et al., 2014; Mas-Herrero \& MarcoPallarés, 2014; Pfabigan et al., 2011; Wu \& Zhou, 2009; also, see Ferdinand, Mecklinger, Kray, \& Gehring, 2012; Oliveira, McDonald, \& Goodman, 2007; Osinsky, Mussel, \& Hewig, 2012).

Interestingly, some studies have shown that cue stimuli themselves can evoke an FRN-like deflection when predicting unfavorable compared to favorable outcomes (Dunning \& Hajcak, 2007; Holroyd, Krigolson, \& Lee, 2011; Liao, Gramann, Feng, Deák, \& Li, 2011; Oliveira, Hickey, \& McDonald, 2014; Osinsky, Mussel, Ohrlein, \& Hewig, 2014; Walsh \& Anderson, 2011; but also see Nieuwenhuis, Schweizer, Mars, Botvinick, \& Hajcak, 2007). Of special importance to the topic investigated here, we recently observed that faces of interaction partners in the so-called Ultimatum Game can evoke an FRN-like deflection, when predicting a subsequent unfair proposal by the partner of how to split a certain amount of money (Osinsky et al. 2014). Thus, early evaluative functions of the pMFC appear to be already engaged by outcome-predictive cues.

Much less attention has been paid to the link between outcome expectations and the response-locked ERN. Independent from objective action correctness, amplitudes in the ERN time range become more negative with increasing subjective error certainty (Pailing \& Segalowitz, 2004; Scheffers \& Coles, 2000) and increasing probability of negative action consequences (e.g., monetary losses; Hewig et al., 2007; Yu \& Zhou, 2009). Thus, an increase of perceived error-probability seems to result in a response-locked ERN-like deflection in situations where the individual does 
not certainly know the actual action correctness at the stage of action execution.

In addition to the abovementioned findings in the EEG time domain, some studies have also investigated how outcome expectancies influence FM $\theta$ activity in the EEG timefrequency domain. ${ }^{1}$ With regard to outcome-locked FM $\theta$, it is still not clear whether it reflects unsigned RPEs (Cavanagh, Figueroa, Cohen, \& Frank, 2012; Hajihosseini \& Holroyd, 2013; Mas-Herrero \& Marco-Pallarés, 2014) or rather signed RPEs (Cavanagh, Frank, Klein, \& Allen, 2010; Cohen et al., 2007). In addition, cue-locked FM $\theta$ activity appears to not differentiate between cues predicting positive and those predicting negative action outcomes (Cavanagh, Eisenberg, Guitart-Masip, Huys, \& Frank, 2013; Cavanagh, ZambranoVazquez, \& Allen, 2012). Moreover, results by Cavanagh, Figueroa, et al. (2012) suggest that response-locked FM $\theta$ power increases with the uncertainty about action outcomes.

\section{The present study}

We aimed to investigate whether the abovementioned electrocortical indices of performance monitoring are sensitive to experience-based expectancies induced by seeing an evaluator's face. To this end, we used a computerized timeestimation task (see Fig. 1) in which participants were asked to estimate the passing of 1 second (Miltner et al., 1997). Each trial of this task mirrored the sequence of events within a typical action-assessment situation from the perspective of the assessed person: first, confrontation with the evaluator; second, performance of the task; and third, reception of the evaluative feedback. Importantly, in each trial, performance was assessed either by a strict evaluator (i.e., who predominantly evaluated performance as incorrect) or a lenient evaluator (i.e., who predominantly evaluated performance as correct). As the evaluator's face was presented before performance and feedback, it could be used as a predictive cue, activating expectations based on the history of prior encounters. In particular, the strict evaluator's face predicts a high error likelihood for the upcoming time estimation and should activate a respective expectation. In contrast, when performance is going to be assessed by the lenient evaluator, participants should expect a positive action outcome. Therefore, the final feedback stimuli in our task could either be expected (i.e., negative feedback given by the strict evaluator and positive feedback given by the lenient evaluator) or unexpected (i.e., negative feedback given by the lenient evaluator and positive feedback given by the strict evaluator).

\footnotetext{
${ }^{1}$ Please note that the ERN and FRN might also be considered as timedomain indices of FM $\theta$. However, for reasons of clarity, when referring to FM $\theta$ we do not include such ERP signatures but only indices derived from time-frequency analyses (e.g., wavelet transformation) of the EEG.
}

We hypothesized that the face-induced outcome expectations should influence monitoring functions of the pMFC at the stages of cue processing (i.e., the faces), action execution (i.e., the button press for time estimation), and outcome processing (i.e., the performance feedbacks). At the stage of cue processing, we expected that the face of the strict evaluator compared to the lenient evaluator should directly evoke more negative ERP amplitudes and higher FM $\theta$ power in the FRN time range (compare, Dunning \& Hajcak, 2007; Holroyd et al., 2011; Liao et al., 2011; Oliveira et al., 2014; Osinsky et al., 2014; Walsh \& Anderson, 2011). At the performance stage, the expectation of higher error likelihood under the supervision of the strict evaluator should result in an ERNlike effect and, again, an increase in FM $\theta$ power (compare, Hewig et al., 2007; Pailing \& Segalowitz, 2004; Scheffers \& Coles, 2000; Yu \& Zhou, 2009). Moreover, this should especially be the case in trials with low performance precision, that is, when task performance strongly deviates from the individual standard. For the feedback stimuli, we expected the typical increase in FRN amplitude and FM $\theta$ power for negative compared to positive performance feedback. As previous research has been inconsistent in whether feedback-locked FRN and FM $\theta$ reflects signed or unsigned RPEs (see above), we had no directed hypotheses in this regard. Finally, visual inspection of the ERP grand-average waves pointed to a negative-going slow amplitude shift following the face of the strict compared to the lenient evaluator at midfrontal sites. This effect resembled the well-known contingent negative variation $(\mathrm{CNV}$; Walter, Cooper, Aldridge, McCallum, \& Winter, 1964), which probably reflects attentional mechanisms of performance preparation (Brunia, van Boxtel, \& Böcker, 2012). As the $\mathrm{CNV}$ has recently received growing attention with regard to its role in outcome anticipation and task-difficulty expectations (e.g., Capa, Bouquet, Dreher, \& Dufour, 2013; Schevernels, Krebs, Santens, Woldorff, \& Boehler, 2014; Silvetti, Nuñez Castellar, Roger, \& Verguts, 2014; van den Berg, Krebs, Lorist, \& Woldorff, 2014), we also conducted an exploratory analysis of this component.

\section{Method}

\section{Participants and general procedure}

Fifty healthy individuals ( 38 female; mean age $=21.6$ years, $S D=3$ ) participated for course credit and additionally received the amount of money they won during the timeestimation task (see below). All participants reported to be free of any mental or neurological disorder and gave written informed consent. ${ }^{2}$ The study was in accordance with the

\footnotetext{
${ }^{2}$ Note that we did not assess participants' handedness. This is a potential limitation of the present study.
} 


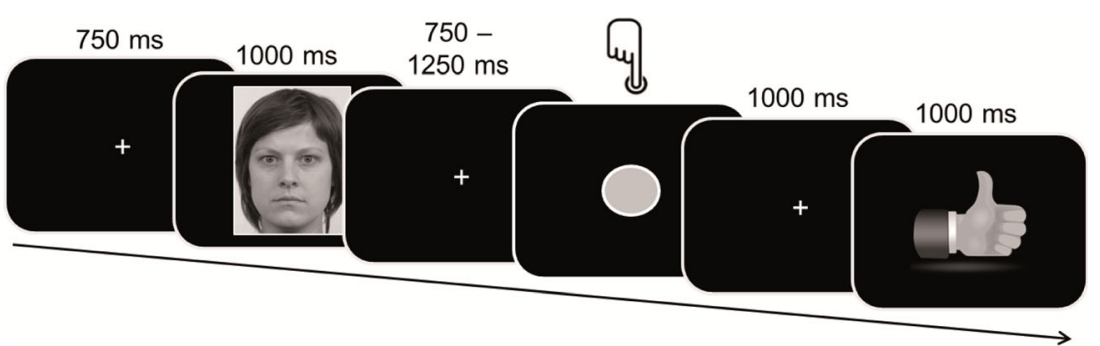

Fig. 1 Schematic depiction of trial events within the used time-estimation task

declaration of Helsinki and was approved by the local ethics committee. After participants arrived at the laboratory, they were thoroughly informed about all measures, and EEG electrodes were prepared. Participants then completed the timeestimation task while EEG was recorded. Following the task, they separately rated each of the two evaluators with regard to unlikability $(1=$ very likable person to $7=$ very unlikable person $)$ and strictness $(1=$ very lenient to $7=$ very strict $)$.

\section{Time-estimation task}

Participants completed a computerized time-estimation task in which their performance was evaluated by two fictional evaluators, one of which was strict whereas the other was lenient. Each trial of this task started with a central fixation cross being presented on the screen for $750 \mathrm{~ms}$. Afterwards, the face of one of the two evaluators appeared in the center of the screen for 1 second. After the face disappeared, a second fixationcross was presented for a random duration of 750 to $1,250 \mathrm{~ms}$. Then, a central gray circle appeared for a fixed duration of 2 seconds. Participants were asked to start time estimation when the gray circle occurs and to press the response button with their right index finger when they felt that 1 second has passed. After the circle disappeared, another fixation-cross was presented before a feedback stimulus appeared for 1 second. Correct time estimation was indicated by a hand with an upward-pointing thumb, whereas an incorrect performance was indicated by a downward-pointing thumb. These stimuli were used because of their well-known positive and negative social meaning, respectively. Nevertheless, participants were thoroughly informed about the feedbacks before the task started. To increase participants' motivation, we told them that 20 cents would be added to their game account for each correct response, whereas they would lose 20 cents for each incorrect response. In the intertrial interval of 1 second, the screen was blank. Before the task started, participants were informed that the face in each trial belongs to the evaluator who will evaluate the subsequent time estimation. Moreover, they were told that one evaluator would be rather strict whereas the other would be more lenient. However, participants were not explicitly informed about the face-strictness contingency but were instead asked to attentively watch the faces and feedbacks because they would be asked to rate the evaluators following the task. Differences in evaluators' assessment style were realized by two adaptive time windows (one for each evaluator), each of which was defined by an upper and lower threshold. Button presses that were given within these time windows were rated as correct, whereas responses outside these windows were rated as incorrect. For each evaluator, the time window had an initial size of $400 \mathrm{~ms}$ around 1 second (i.e., 800-1,200 ms). After a correct response the size of the window for the next encounter was decreased by $10 \mathrm{~ms}$ for the lenient evaluator and $30 \mathrm{~ms}$ for the strict evaluator. When performance was incorrect, the window was increased by $30 \mathrm{~ms}$ for the lenient evaluator and $10 \mathrm{~ms}$ for the strict evaluator. These differences in performancebased criteria adjustments lead to a mean incorrect rate of $74 \%$ (range $69 \%-84 \%, S D=2 \%$ ) for the strict evaluator and $24 \%$ (range $17 \%-30 \%, S D=2 \%$ ) for the lenient evaluator. Thus, positive feedback was more frequent and, consequently, expected for the lenient evaluator and less frequent and unexpected for the strict evaluator. In contrast, negative feedback was more frequent and expected for the strict evaluator and less frequent and unexpected for the lenient evaluator. The whole task consisted of 200 trials, 100 for each evaluator. Trials were presented in random order, with the only restriction that performance was not assessed by the same evaluator more than five times in a row. To consider the generation of expectations, Trials 1 to 10 for each evaluator were discarded from analyses. Evaluators' and participants' gender was always identical, and the face-strictness assignment was counterbalanced across participants. Face stimuli were taken from the Radboud face database (Langner et al., 2010). All stimuli were presented on a 21 -inch screen $(60 \mathrm{~Hz}$ refresh rate) with a black background. The task was controlled by Presentation experimental software (Neurobehavioral Systems Inc., Albany, CA).

\section{EEG recordings and data analyses}

During task performance, we recorded EEG (recording band pass: $0.016-80 \mathrm{~Hz}$, sampling rate: $250 \mathrm{~Hz}$ ) from $31 \mathrm{Ag} / \mathrm{AgCl}$ electrodes (Fp1, Fp2, F9, F7, F3, Fz, F4, F8, F10, FC5, FC1, FCz, FC2, FC6, T7, C3, C4, T8, TP9, CP1, CP2, TP10, P7, P3, Pz, P4, P8, PO9, O1, O2, and PO10) mounted on the scalp with an elastic cap (Easy Cap GmbH, Herrsching, Germany), 
using a BrainAmp amplifier (Brain Products $\mathrm{GmbH}$, Gilching, Germany). Recordings sites were prepared so that impedances were below $10 \mathrm{k} \Omega$. Online reference was placed at position $\mathrm{Cz}$. For offline data processing, Brain Vision Analyzer 2.0 (Brain Products $\mathrm{GmbH}$ ) software was used. First, data were re-referenced to the average of electrodes TP9 and TP10 (mastoids), and the former reference Cz was reinstated as an additional data channel. Data were then filtered using a $0.1 \mathrm{~Hz}$ high-pass (Butterworth Zero Phase Filter, $3 \mathrm{db}$ point, $24 \mathrm{~dB} /$ oct roll-off) and a $20 \mathrm{~Hz}$ low-pass filter (Butterworth Zero Phase Filter, $3 \mathrm{db}$ point, $48 \mathrm{~dB} /$ oct rolloff). Afterwards, data were corrected for ocular artifacts using an Independent Component Analysis based correction method implemented in the Brain Vision Analyzer. Except for analyses of the CNV slow wave, an additional high-pass filter of $1 \mathrm{~Hz}$ (Butterworth Zero Phase Filter, $3 \mathrm{db}$ point, $48 \mathrm{~dB} /$ oct rolloff) was applied. This was done to reduce potential distortions due to the low-frequent and positive-going P300 component, which temporally overlaps, especially with the FRN and has also been shown to be influenced by outcome valence and expectancies (e.g., Osinsky et al., 2012). Afterwards, data were segmented as follows. For time-frequency analysis of FM $\theta$, we built 2,000 ms epochs around events of interest (1,000 to $1,000 \mathrm{~ms}$; face onset, responses, and feedback onset). For analyses of the face-locked and feedback-locked FRN, 1, $000 \mathrm{~ms}$ epochs (-200 to $800 \mathrm{~ms}$ ) were used. Epochs for the response-locked ERN were $900 \mathrm{~ms}$ long (-400 to $500 \mathrm{~ms}$ ). Finally, for analyses of the face-locked CNV, epochs started $200 \mathrm{~ms}$ before and ended $1,750 \mathrm{~ms}$ following face onset. Epochs were excluded from further analyses when the following criteria were met at any of the EEG channels: maximal voltage step of $>20 \mu \mathrm{V} / \mathrm{ms}$; maximal voltage difference $>150 \mu \mathrm{V}$ within the segment. For analyses in the time domain (ERN, FRN, and CNV), segments were averaged and baseline corrected (ERN: -400 to $-200 \mathrm{~ms}$; FRN and CNV: -200 to $0 \mathrm{~ms}$ ) for each participant and condition of interest. After visual inspection of grand average waves, the FRN was quantified as mean amplitude 220 to $300 \mathrm{~ms}$ following feedback onset or face onset. With regard to response-locked ERP data, grand average waves pointed to potential effects already in the preresponse phase. We therefore quantified the ERN mean amplitude for two time windows, one from -160 to $0 \mathrm{~ms}$ and the other from 0 to $100 \mathrm{~ms}$. For CNV analyses, we calculated mean amplitudes 1,000 to $1,750 \mathrm{~ms}$ after face onset. For timefrequency analyses, segmented data were transformed, using a family of complex Morlet wavelets from 1 to $20 \mathrm{~Hz}$ in linear steps of $1 \mathrm{~Hz}$. The complex Morlet wavelets are defined as Gaussian-windowed complex sine functions: $w\left(t, f_{o}\right)=A e$ $\left(\frac{-t^{2}}{2 \sigma_{t}^{2}}\right) e^{\left(2 i \pi f_{o} t\right)}$, with $\sigma_{t}=\frac{1}{2} \pi \sigma_{f}$ and $A=\left(\sigma_{t} \sqrt{\pi}\right)^{-1 / 2}$, the latter resulting in total energy of 1 for all frequency levels (Tallon-Baudry, Bertrand, Peronnet, \& Pernier, 1998). Constant ratio $\left(f_{o} / \sigma_{f}\right)$ was set 6.7 (compare, Hajihosseini \&
Holroyd, 2013; Marco-Pallarés et al., 2008). For each frequency layer, power values were baseline corrected by subtracting the mean activity in the time window -500 to $-250 \mathrm{~ms}$ before feedback onset from each data point. Segments were averaged for each participant and condition of interest. For statistical analyses, FM $\theta$ mean power 4 to $8 \mathrm{~Hz}$ was extracted from 200 to $400 \mathrm{~ms}$ following the faces and feedbacks. Based on visual inspection of time-frequency plots, we analyzed two time windows for response-locked FM $\theta$ power, one from 0 to $200 \mathrm{~ms}$ and a later one from 400 to $600 \mathrm{~ms}$.

Data for electrodes $\mathrm{Fz}, \mathrm{FCz}$, and $\mathrm{Cz}$ were statistically analyzed using repeated-measures analyses of variance (ANOVA), pairwise $t$ tests (uncorrected for multiple comparisons), and nonparametric Wilcoxon signed-rank test as implemented in the SPSS software package (IBM, Armonk, NY, USA). In case of violation of sphericity assumption, Greenhouse-Geisser correction was applied and epsilon $(\varepsilon)$ is reported. All tests were two-tailed, and $p$ values $\leq .05$ were considered significant. Partial eta square $\left(\eta^{2}\right)$ is reported as effect-size measure for significant effects in the ANOVAs.

\section{Results}

\section{Performance data and evaluator ratings}

Individual mean response times under the supervision of the strict evaluator (mean $=986 \mathrm{~ms}, S D=160$ ) and lenient evaluator (mean $=986 \mathrm{~ms}, S D=169$ ) did not significantly differ, $t(49)=0.03, p=.975$. In addition, we compared the two contexts with regard to the absolute deviations of the individual mean estimation latencies from 1 second. As these scores were not normally distributed and had a strong positive skewness $(>2.98)$, we used the nonparametric Wilcoxon signedrank test. Under supervision of the strict evaluator (median = $69 \mathrm{~ms}$ ) the absolute deviation from 1 second was significantly smaller $(Z=-2.09, p=.037)$ than under supervision of the lenient evaluator (median $=78 \mathrm{~ms}$ ). Thus, time estimation was more precise when it was going to be assessed by the strict evaluator.

Analyses of posttask ratings (possible range 1 to 7) revealed that the strict evaluator was rated as being stricter, mean $=5.66, S D=0.87, t(49)=13.28, p<.001$, and more unlikeable, mean $=4.62, S D=1.28, t(49)=2.88, p=.006$, compared to the lenient evaluator, strictness: mean $=2.78, S D$ $=0.82$, unlikability: mean $=3.74, S D=1.31$.

\section{Face-locked EEG results}

Grand average ERP waves as well as time-frequency plots for the face-locked data are shown in Fig. 2. Descriptive statistics are presented in Table 1. The electrocortical indices of interest (i.e., FRN, FM $\theta$ power, and CNV) were analyzed using a $3 \times$ 
a $\mathrm{FCz}$

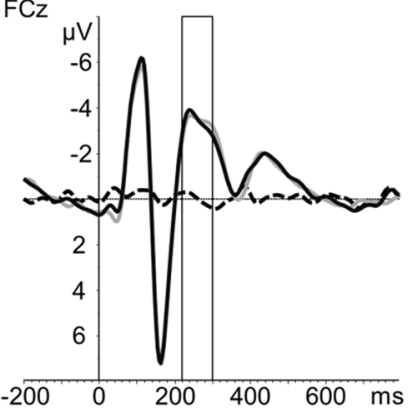

b

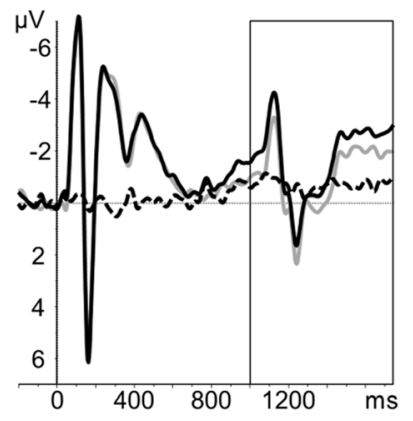

C
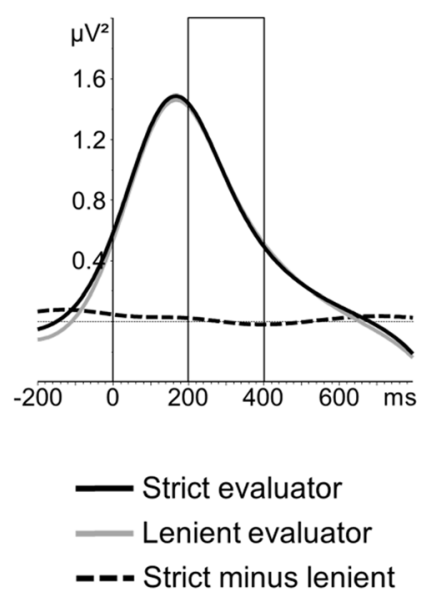

Strict minus lenient 220-300 ms

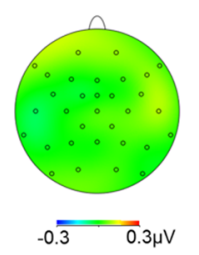

Strict minus lenient $1000-1750 \mathrm{~ms}$

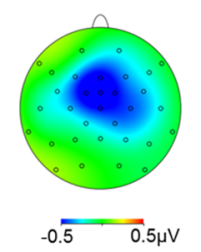

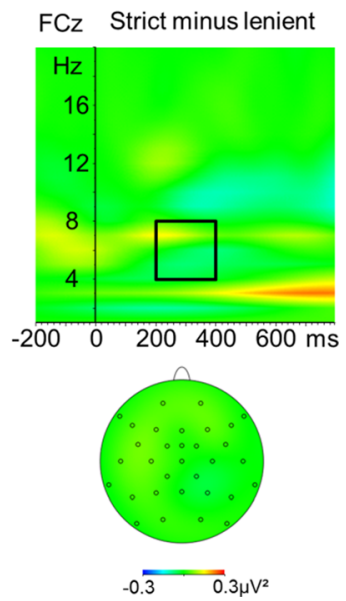

Fig. 2 Grand average ERP plots and time-frequency plots for the effect of evaluator's strictness on face-locked EEG data. Rectangles mark the time- and time-frequency windows that were used for statistical analyses and generation of the topographical maps. All ERP and time-frequency plots refer to electrode FCz. a In the time range of the FRN (220-300 ms), no amplitude difference occurred between the two faces. b A negativegoing, sustained amplitude shift followed the face of the strict compared to the lenient evaluator at fronto-central sites $(1,000-1,750 \mathrm{~ms}) . \mathbf{c}$ Like for the FRN analyses, no face-related difference occurred for the FM $\theta$ response (4-8 Hz, 200-400 ms)

2 repeated-measures ANOVA design with the within-subject factors "electrode" (Fz, FCz, Cz) and "evaluator's strictness" (strict, lenient).

Face-locked FRN amplitude The main effect of "electrode" was significant, $F(2,98)=5.42, p=.015, \eta_{\mathrm{p}}^{2}=.10, \varepsilon=.66$,

Face-locked FM $\theta$ power The main effect of "electrode" was significant, $F(2,98)=13.15, p=.015, \eta^{2}=.21, \varepsilon=.73$, reflecting lower FM $\theta$ power at $\mathrm{Fz}$ compared to $\mathrm{FCz}, t(49)=$ $4.84, p<.001$, and $\mathrm{Cz}, t(49)=2.98, p=.004$, whereas there was no significant difference between $\mathrm{FCz}$ and $\mathrm{Cz}, t(49)=$ $1.88, p=.071$. Neither the main effect of "evaluator's strictness" $F(1,49)=0.01, p=.953$, nor the interaction with "electrode" $F(2,98)=0.11, p=.831, \varepsilon=.72$, reached statistical significance. Thus, we did not observe the hypothesized FM $\theta$ power increase in direct response to the strict evaluator's face.

with pairwise comparisons showing that amplitudes were less negative at $\mathrm{Cz}$ compared to $\mathrm{Fz}, t(49)=2.44, p=.018$, and $\mathrm{FCz}, t(49)=2.93, p=.005$, whereas there was no significant difference between $\mathrm{FCz}$ and $\mathrm{Cz}, t(49)=1.12, p=.270$. As can be clearly seen in Fig. 2, there was no substantial amplitude difference between the face conditions in the FRN time range. Accordingly, both the main effect of "evaluator strictness," $F(1,49)=.01, p=.926$, and the interaction of "electrode" $\times$ "evaluator strictness" $F(2,98)=1.33, p=.265, \varepsilon=.69$, were far from statistical significance. Thus, we did not observe the expected cue-FRN effect for the evaluator faces.

Face-locked CNV amplitude The main effect of "electrode" was significant, $F(2,98)=19.02, p<.001, \eta_{\mathrm{p}}^{2}=.28, \varepsilon=.87$, and pairwise comparisons showed that amplitudes were generally more negative at electrode $\mathrm{FCz}$ compared to electrode $\mathrm{Fz}, t(49)=6.58, p<.001$, and $\mathrm{Cz}, t(49)=2.17, p=.035$, and at electrode $\mathrm{Cz}$ compared to $\mathrm{Fz}, t(49)=3.50, p=.001$. Moreover, the main effect of "evaluator's strictness" was significant, $F(1,49)=6.60, p=.013, \eta^{2}{ }_{p}=.12$, reflecting more negative amplitudes following the face of the strict compared to the lenient evaluator. The interaction of "electrode" $\times$ "evaluator's strictness" was not significant, $F(2,98)$ $=0.70, p=.465, \varepsilon=.77$. Thus, in the time window between face offset and the imperative stimulus (i.e., the circle that starts time estimation) we observed a slow, negative-going amplitude shift at fronto-central scalp ${ }^{3}$ sites when the face predicted a high error likelihood.

\section{Response-locked EEG results}

Figure 3 shows grand average waves and time-frequency plots for the response-locked data. Means and standard deviations

\footnotetext{
${ }^{3}$ Figure $2 \mathrm{~b}$ suggests that the $\mathrm{CNV}$ effect might be more pronounced over the right compared to the left hemisphere. However, when including laterality as an additional factor (i.e., by including electrodes F3, FC1, andC3 for the left hemisphere and F4, FC2, and C4 for the right hemisphere) the main effect of "evaluator's strictness" remained significant, $F(1,49)=5.09, p=.029$, whereas we did not observe a significant interaction of "evaluator's strictness" × "laterality" $F(2,98)=1.47, p=$ .24 .
} 
Table 1 Means and standard deviations (in brackets) for ERP amplitudes (in $\mu \mathrm{V}$ ) and FM $\theta$ power (in $\mu \mathrm{V}^{2}$ ) in the time windows of interest across electrodes $\mathrm{Fz}, \mathrm{FCz}$, and $\mathrm{Cz}$

\begin{tabular}{|c|c|c|c|c|}
\hline Face locked & Strict evaluator & Lenient evaluator & & \\
\hline ERP $220-300 \mathrm{~ms}$ & $-3.34(3.40)$ & $-3.37(3.56)$ & & \\
\hline ERP $1000-1750 \mathrm{~ms}$ & $-1.23(2.85)$ & $-0.65(2.79)$ & & \\
\hline FM $\theta 200-400 \mathrm{~ms}$ & $0.89(0.95)$ & $0.89(0.91)$ & & \\
\hline Response locked & Strict evaluator / high precision & Strict evaluator / low precision & Lenient evaluator / high precision & Lenient evaluator / low precision \\
\hline ERP $-160-0 \mathrm{~ms}$ & $-1.40(1.84)$ & $-0.57(1.48)$ & $-1.13(1.53)$ & $-0.52(1.51)$ \\
\hline ERP 0-100 ms & $-2.63(2.35)$ & $-2.20(1.91)$ & $-2.37(1.80)$ & $-2.03(2.07)$ \\
\hline FM $\theta 0-200 \mathrm{~ms}$ & $0.18(0.56)$ & $0.21(0.43)$ & $0.10(0.63)$ & $0.07(0.54)$ \\
\hline FM $\theta 400-600 \mathrm{~ms}$ & $0.16(0.84)$ & $0.04(0.78)$ & $0.16(0.81)$ & $-0.06(0.59)$ \\
\hline Feedback locked & Unexpected negative & Expected negative & Unexpected positive & Expected positive \\
\hline ERP $220-300 \mathrm{~ms}$ & $0.23(5.08)$ & $1.12(4.31)$ & $4.86(5.27)$ & $4.65(4.62)$ \\
\hline FM $\theta 200-400 \mathrm{~ms}$ & $2.78(1.70)$ & $2.33(1.36)$ & $1.57(1.54)$ & $1.38(1.05)$ \\
\hline
\end{tabular}

are presented in Table 1. As the response-locked ERN and FM $\theta$ response in our task may not only depend on faceinduced expectancies but also on performance precision, we also considered the latter factor. For this purpose, when calculating mean ERN amplitude and FM $\theta$ power, we distinguished between trials with high individual time estimation precision (25th to 75th individual percentile) and those with low precision (below the 25th percentile or above the 75th

a

$\mathrm{Fz} \mu \mathrm{V}$

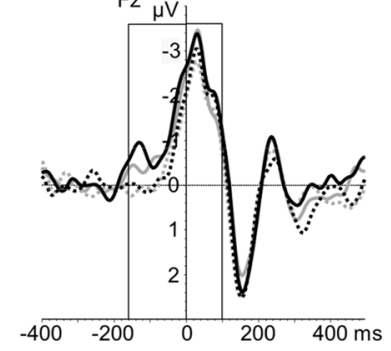

- Strict evaluator/high precision

Lenient evaluator/high precision

..... Strict evaluator / low precision

".... Lenient evaluator/low precision

b

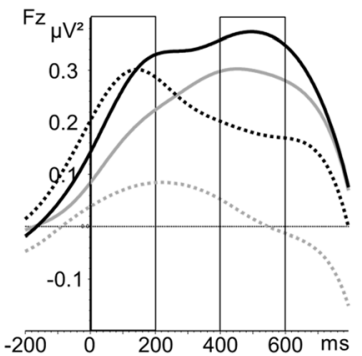

- Strict evaluator/ high precision

_ Lenient evaluator/high precision

..... Strict evaluator / low precision

...... Lenient evaluator/low precision

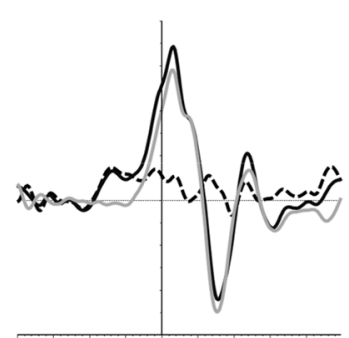

- High precision

- Low precision

- - High minus low

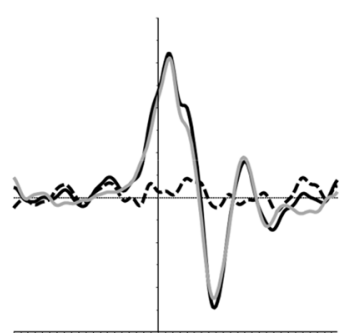

- Strict evaluator

Lenient evaluator

- - Strict minus lenient
High minus low precision

$-160-0 \mathrm{~ms} \quad 0-100 \mathrm{~ms}$

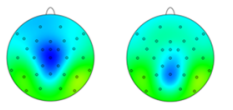

Strict minus lenient

$-160-0 \mathrm{~ms} \quad 0-100 \mathrm{~ms}$

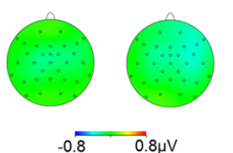

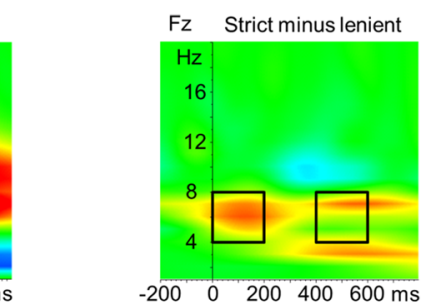
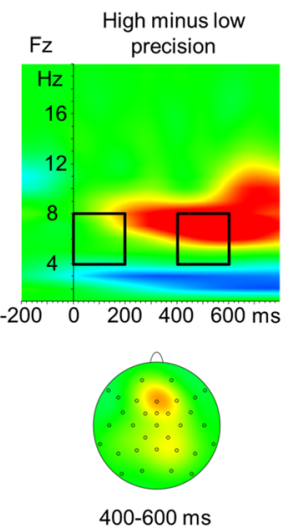

$-0.3-3 \mu V^{2}$

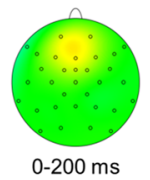

central negative-going amplitude shift -160 to $0 \mathrm{~ms}$ before the response and an increased FM $\theta$ power 400 to $600 \mathrm{~ms}$ following the response. In addition, when performance was going to be assessed by the strict compared to the lenient evaluator, we observed a postresponse ( 0 to $200 \mathrm{~ms}$ ) increase in $\mathrm{FM} \theta$ power 
percentile) separately for the strict and lenient condition. Consequently, ERN and FM $\theta$ power were analyzed using 3 $\times 2 \times 2$ repeated-measures ANVOA design with the withinsubject factors "electrode" (Fz, FCz, and $\mathrm{Cz}$ ), "evaluator's strictness" (strict, lenient), and "time estimation precision" (low, high).

Response-locked ERN amplitude $\mathbf{- 1 6 0}$ to $0 \mathrm{~ms}$ The main effect of "electrode" was highly significant, $F(2,98)=$ $17.57, p<.001, \eta_{\mathrm{p}}^{2}=.26, \varepsilon=.68$, reflecting more negative amplitudes at $\mathrm{FCz}$ and $\mathrm{Cz}$ compared to $\mathrm{Fz}, \mathrm{FCz}$ vs. Fz: $t(49)=$ $5.30, p<.001 ; \mathrm{Cz}$ vs. Fz: $t(49)=4.26, p<.001 ; \mathrm{Cz}$ vs. FCz: $t(49)=1.22, p=.229$. More importantly, the main effect of "time estimation precision" was significant, $F(1,49)=5.92, p$ $=.019, \eta_{\mathrm{p}}^{2}=.11$, reflecting more negative amplitudes when performance precision was high compared to low (see Figure 3a). This effect was further modulated my the "electrode" factor, $F(2,98)=5.61, p=.011, \varepsilon=.72$, being larger at $\mathrm{FCz}, t(49)=3.15, p=.003$, and $\mathrm{Cz}, t(49)=2.53$, $p=.015$, compared to Fz. None of the effects involving the "evaluator's strictness" factor reached statistical significance, main effect: $F(1,49)=1.89, p=.175$; "evaluator's strictness" $\times$ "electrode": $F(2,89)=0.91, p=.364, \varepsilon=.61$; "evaluator's strictness" $\times$ "time estimation precision": $F(1,49)=0.57$, $p=.45$; "evaluator's strictness" $\times$ "time estimation precision" $\times$ "electrode": $F(2,89)=0.01, p=.968, \varepsilon=.73$. In sum, while there was no effect of the evaluator context on fronto-medial ERP amplitudes -160 to $0 \mathrm{~ms}$, more accurate performance was generally associated with more negative amplitudes in this preresponse time window.

Response-locked ERN amplitude 0 to $100 \mathrm{~ms}$ The main effect of "electrode" was highly significant, $F(2,98)=$ 19.32, $p<.001, \eta_{\mathrm{p}}^{2}=.28, \varepsilon=.67$. Pairwise comparisons showed that amplitudes were most negative at $\mathrm{FCz}, \mathrm{FCz}$ vs. Fz: $t(49)=4.38, p<.001 ; \mathrm{Cz}$ vs. Fz: $t(49)=2.48, p=.017 ; \mathrm{Cz}$ vs. FCz: $t(49)=6.71, p<.001$. None of the other effects reached statistical significance, main effect "time estimation precision": $F(1,49)=2.73, p=.11$; main effect "evaluator's strictness": $F(1,49)=1.37, p=.25$; "time estimation precision" $\times$ "electrode": $F(2,89)=2.99, p=.075, \varepsilon=.69$; "evaluator's strictness" × "electrode": $F(2,89)=0.15, p=.78, \varepsilon=.68$; "time estimation precision" $\times$ "evaluator's strictness": $F(1,49)=0.10$, $p=.76$; "time estimation precision" $\times$ "evaluator's strictness" $\times$ "electrode": $F(2,89)=1.93, p=.16, \varepsilon=.74$. Thus, ERN amplitude following the response was insensitive to evaluator's strictness and time estimation precision.

Response-locked FM $\theta$ power 0-200 ms Importantly, the main effect of "evaluator's strictness" was significant, $F(1$, $49)=5.48, p=.023, \eta_{\mathrm{p}}^{2}=.1$, reflecting higher FM $\theta$ power when time estimation was going to be assessed by the strict compared to the lenient evaluator (see Figure $3 b$ ). The interaction of "electrode" $\times$ "evaluator's strictness" also reached significance, $F(2,98)=5.63, p=.012, \eta^{2}{ }_{p}=.1, \varepsilon=$ .70. Post hoc pairwise comparisons revealed that the difference between the two evaluator conditions was significant at electrode $\mathrm{Fz}, t(49)=2.67, p=.010)$ and $\mathrm{FCz}, t(49)=2.53, p=$ .015 , but not at $\mathrm{Cz}, t(49)=1.03, p=.307$. None of the other effects was significant, main effect "electrode": $F(1,49)=$ $3.28, p=.061$; main effect "time estimation precision": $F(1$, $49)=0.01, p=.998$; "time estimation precision" $\times$ "electrode": $F(2,89)=0.92, p=.387, \varepsilon=.83$; "time estimation precision" × "evaluator's strictness": $F(1,49)=0.46, p=.499$; "time estimation precision" $\times$ "evaluator's strictness" $\times$ "electrode": $F(2,89)=1.75, p=.188, \varepsilon=.74$. Thus, FM $\theta$ power in the first $200 \mathrm{~ms}$ following the response was sensitive to face-induced expectancies but not to performance precision.

Response-locked FM $\theta$ power $400-600$ ms For the late time window, we observed a significant effect of "time estimation precision", reflecting higher FM $\theta$ power when performance precision was high compared to low, $F(1,49)=4.07, p=.049$, $\eta_{\mathrm{p}}^{2}=.08$. In addition, the main effect of electrode was also significant, $F(2,98)=9.63, p<.001, \eta_{\mathrm{p}}^{2}=.16, \varepsilon=.67$. Pairwise comparisons showed that FM $\theta$ power was higher at $\mathrm{Fz}$ compared to $\mathrm{FCz}$ and $\mathrm{Cz}, \mathrm{Fz}$ vs. FCz: $t(49)=4.27, p<$ .001 ; Fz vs. Cz: $t(49)=3.13, p=.003$; Cz vs. FCz: $t(49)=$ $0.78, p=.44$. None of the other effects reached the level of statistical significance, main effect "evaluator's strictness": $F(1,49)=0.69, p=.409$; "evaluator's strictness" $\times$ "electrode": $F(2,89)=3.05, p=.081, \varepsilon=.58$; "time estimation precision" $\times$ "electrode": $F(2,89)=1.65, p=.205$, $\varepsilon=.66$; "time estimation precision" $\times$ "evaluator's strictness": $F(1,49)=0.78, p=.380$; "time estimation precision" $\times$ "evaluator's strictness" $\times$ "electrode": $F(2,89)=0.18$, $p=.73, \varepsilon=.63$. Hence, in the late time window, 400-600 ms following the response, FM $\theta$ was sensitive to performance precision but insensitive to face-induced expectancies.

\section{Feedback-locked EEG results}

For the feedback-locked data, grand average waves and timefrequency plots are shown in Fig. 4. Descriptive statistics are listed in Table 1. FRN amplitude (220-300 ms) and FM $\theta$ power (200-400 ms) were analyzed with a $3 \times 2 \times 2$ repeated-measures ANOVA design with the within-subject factors "electrode" (Fz, FCz, Cz), "feedback valence" (positive, negative), and "feedback expectancy" (expected, unexpected).

Feedback-locked FRN amplitude The main effect of "electrode" was significant, $F(2,98)=29.15, p<.001, \eta^{2}$ p $=.37, \varepsilon=.57$. Amplitudes in the FRN time range were generally more positive at electrode $\mathrm{Cz}$ compared to $\mathrm{FCz}, t(49)=$ $3.23, p=.002$, and Fz, $t(49)=5.47, p<.001$, and at $\mathrm{FCz}$ 


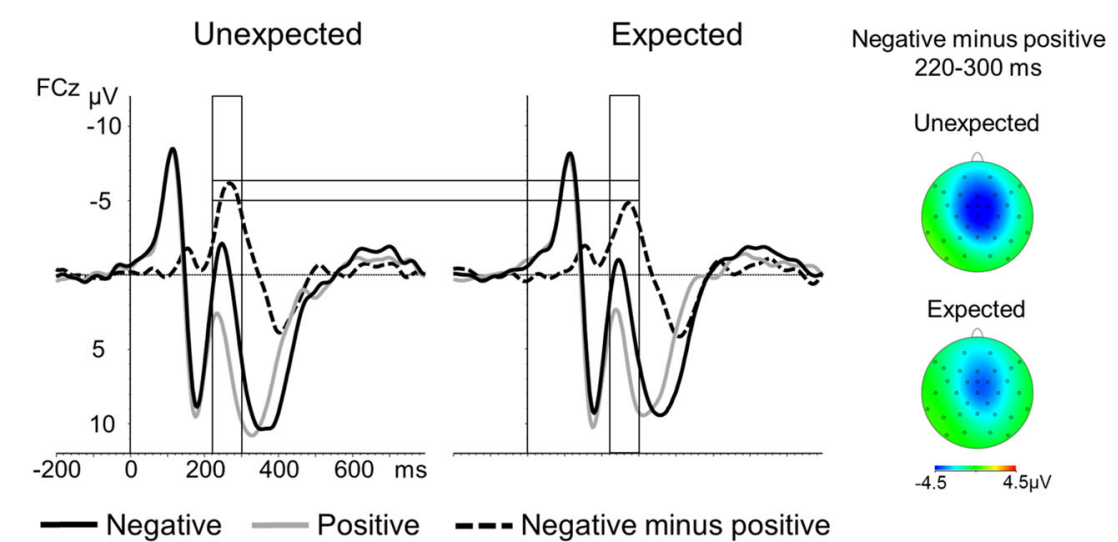

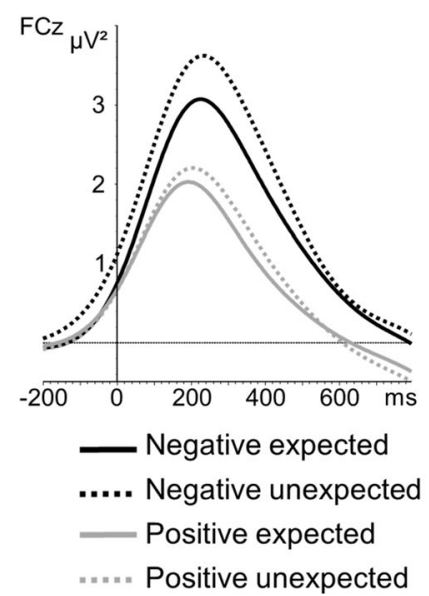

Fig. 4 Grand-average ERP plots and time-frequency plots for the feedback-locked EEG data as a function of outcome valence and expectancy. Rectangles mark the time- and time-frequency windows that were used for statistical analyses and generation of the topographical maps. All ERP and time-frequency plots refer to electrode FCz. a Negative compared to positive outcomes evoked more
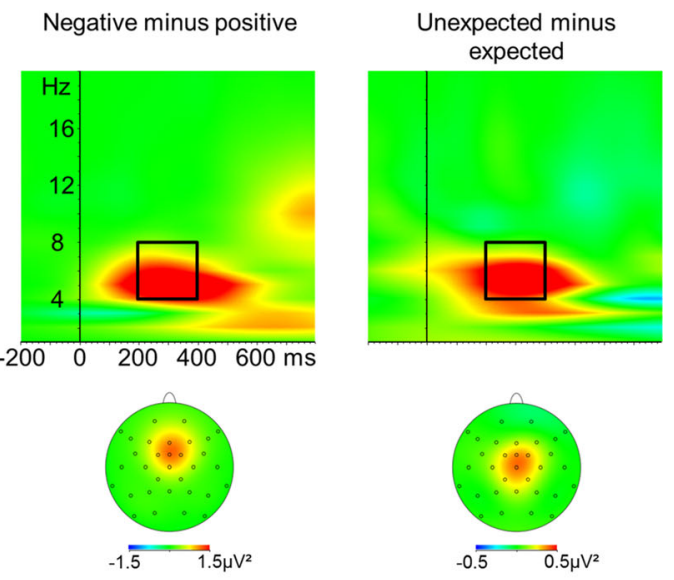

negative amplitudes in the FRN time range (220-300 ms) and this effect was higher for unexpected compared to expected feedback (see horizontal lines between the graphs). b FM $\theta$ power $(4-8 \mathrm{~Hz}, 200-400 \mathrm{~ms}$ ) was higher for negative compared to positive feedback and, independent of outcome valence, higher for unexpected to expected feedback

comparisons indicated that this effect was mainly driven by more negative FRN amplitudes following unpredicted compared to predicted negative feedbacks, $t(49)=2.56, p=.014$. In contrast, we observed no significant FRN difference between expected and unexpected positive outcomes, $t(49)$ $=0.58, p=.562$. No other effect in the ANOVA reached statistical significance (all $F_{\mathrm{s}}<2.65$, all $p \mathrm{~s}>.102$ ). In sum, we observed the typical FRN difference between negative and positive performance feedback which was more pronounced for unexpected compared to expected outcomes. This latter expectancy modulation was mainly driven by an FRN increase when performance feedback was worse than expected (i.e., negative feedback given by the lenient evaluator).

Feedback-locked FM $\theta$ power Feedback-locked FM $\theta$ power was generally higher at electrode $\mathrm{FCz}$ compared to $\mathrm{Cz}$ and $\mathrm{Fz}$, as indicated by a significant main effect of "electrode" and pairwise comparisons, $F(2,98)=27.81, p<.001, \eta_{\mathrm{p}}^{2}=.36$, 
$\varepsilon=.75 ; \mathrm{FCz}$ vs. Fz: $t(49)=7.29, p<.001$; FCz vs. Cz: $t(49)$ $=8.02, p<.001$; Cz vs. Fz: $t(49)=0.30, p=.769$. A significant main effect of "feedback valence" reflected higher FM $\theta$ power for negative compared to positive feedback, $F(1,49)$ $=40.00, p<.001, \eta_{\mathrm{p}}^{2}=.42$. This effect differed between electrode sites as indicated by a significant interaction of "electrode" $\times$ "feedback valence", $F(2,98)=10.66$, $p<.001, \eta_{\mathrm{p}}^{2}=.18, \varepsilon=.72$. Pairwise comparisons revealed that the valence effect was larger at FCz compared to Fz, $t(49)$ $=2.86, p=.006$, and $\mathrm{Cz}, t(49)=5.76, p<.001$, whereas there was no significant difference between $\mathrm{Fz}$ and $\mathrm{Cz}, t(49)=1.70$, $p=.095$. Importantly, we also observed a significant main effect of "feedback expectancy", $F(1,49)=7.23, p=.010$, $\eta_{\mathrm{p}}^{2}=.13$, which indicated that FM $\theta$ was generally higher for unexpected compared to expected feedbacks. This effect was also modulated by electrode site, $F(2,98)=6.06, p=.010, \eta^{2}$ $=.11, \varepsilon=.67$, with pairwise comparisons showing that it was smaller at Fz compared to $\mathrm{FCz}, t(49)=3.48, p=.001$, and $\mathrm{Cz}$, $t(49)=2.21, p=.031$, whereas there was no difference between FCz and $\mathrm{Cz}, t(49)=0.40, p=.690$. Neither the two-way interaction of "feedback valence" $\times$ "feedback expectancy" nor the three-way interaction of "feedback valence" $x$ "feedback expectancy" $\times$ "electrode" reached statistical significance (all $F_{\mathrm{s}}<1.44$, all $p \mathrm{~s}>$.240). In sum, we observed the typical pattern of higher FM $\theta$ power after negative compared to positive feedback. In addition, FM $\theta$ power was generally increased when performance feedback was unexpected, independent of feedback valence.

\section{Discussion}

Several prior studies have demonstrated that simple visual cue stimuli that are predictive of action outcomes influence neural mechanisms of performance-monitoring measured by EEG. Here, we aimed to extend this literature by using more ecologically valid cues, that is, facial stimuli. In daily life, evaluative feedback by others is often of eminent importance, informing us whether our behavior fits particular standards or criteria. Therefore, it is likely that faces of known evaluators also act as predictive cues that are used for adaptations in performance-monitoring functions of the pMFC. In the present study we tested this assumption by analyzing prominent electrocortical signatures of performance monitoring (i.e., the ERN, the FRN, and FM $\theta$ ) and task anticipation (i.e., the $\mathrm{CNV}$ ). For this purpose we applied a simple time-estimation task in which individuals were assessed by a fictive strict evaluator and a lenient evaluator. Our EEG analyses were guided by the question of whether evaluators' faces can act as predictive cues, activating experience-based expectancies about action evaluation which then influence neural functions of performance monitoring and preparation at the stages of cue processing, task performance, and feedback reception.
Our behavioral results clearly show that participants learned to distinguish between the two evaluators based on their experiences throughout the task. While the time estimation was more precise on average under the supervision of the strict evaluator, he or she was explicitly rated more negative than the lenient evaluator. These findings confirm that participants registered the face-performanceoutcome contingencies, what is a prerequisite for the assumed relations between face-activated expectancies and electrocortical indices of performance monitoring. In the following, we will discuss our EEG findings according to the order of events within a single trial.

\section{Cue-processing stage}

Concerning the face-locked data, we could not detect a substantial difference in FRN amplitude and FM $\theta$ power between the two evaluator conditions briefly following the onset of the face stimuli. These negative findings indicate that face-induced expectancies in our task do not influence early evaluative performance monitoring functions of the pMFC at the stage of cue processing. This is, at first glance, inconsistent with several prior studies reporting an FRN-like effect shortly following the onset of outcome-predictive cues (Dunning \& Hajcak, 2007; Liao et al., 2011; Osinsky et al., 2014; Walsh \& Anderson, 2011). A closer look, however, reveals important methodological differences between the behavioral tasks used in these studies and the time-estimation paradigm employed here. In the studies by Liao et al. (2011) and Walsh and Anderson (2011), in each single trial the cue stimulus followed an initial behavioral choice and was therefore per se a choice outcome. In the studies by Dunning and Hajcak (2007) and Osinsky et al. (2014), the negative cues that evoked an FRN-like deflection perfectly predicted the most unfavorable task outcome. Thus, the negative cues in these four studies were not only predictive of the final unfavorable outcome but are likely to have been interpreted as kind of negative outcomes themselves. This was probably different in the present study, as in each single trial of our time-estimation task the face cue was presented before any action and had no perfect predictive value with regard to the trial outcome. Interestingly, similar cue characteristics have been used by Nieuwenhuis et al. (2007), who also found no cuelocked effects in the FRN time range. Altogether, this implies that early evaluative functions of the pMFC may not be sensitive to probabilistic (i.e., nondeterministic) information about action outcomes before any action has been executed. However, this does not necessarily mean that the pMFC is generally insensitive to such information. Indeed, our own results suggest that the pMFC may distinguish between positive and negative cues at later 
stages of stimulus processing. More precisely, in the time window between the face cue and the imperative stimulus (i.e., the stimulus that starts the time estimation), we observed a slow negative-going amplitude shift when the face predicted a strict compared to a lenient evaluation rule. This effect resembled the well-known CNV and had a fronto-central distribution, what is in accordance with a potential source in the pMFC. The CNV has been interpreted to reflect an anticipatory preparation of neural mechanisms which are later involved in task processing (Brunia et al., 2012). The CNV-like effect in our study may therefore reflect anticipatory control processes of the pMFC, monitoring functions of which are later engaged during the stages of task performance and feedback reception. Interestingly, similar effects have been observed in some other recent studies. Oliveira et al. (2014) observed a fronto-central negative-going deflection after cues that signaled a high difficulty of the upcoming task. While they interpreted this deflection as an FRN-like signature, the late timing and obviously sustained duration of this effect was rather untypical for an FRN (see Fig. 3a in Oliveira et al., 2014) and more resembled a CNV. In another study, Schevernels et al. (2014) also observed a CNV after cues that signaled that the following task will be difficult and rewarded. Similar results were found by van den Berg et al. (2014), who reported a CNV effect in response to cues that indicated that individuals would later have to react to a Stroop stimulus (i.e., a color word that could be written in a congruent color or incongruent color), especially when task performance was going to be rewarded. As incongruent Stroop stimuli are known to engage performance monitoring functions of the pMFC (Carter \& van Veen, 2007), the results by van den Berg et al. also support the notion that the mentioned CNV effects may reflect an anticipatory preparation of performance-monitoring functions of the pMFC when these are likely to be needed later on. It should also be noted, however, that Aarts and colleagues (2008) found a general increase in pMFC activity (measured by functional magnetic resonance imaging) in response to both high-error-likelihood cues and low-errorlikelihood cues when comparing these stimuli to uninformative cues. They interpreted this finding to show that cue-related activity of the pMFC is linked to general anticipatory control. Future studies may further address this issue by expanding the experimental design of the present study by including an additional, more neutral evaluator (i.e., one who assesses performance as correct and incorrect with equal probability). At the moment, we can conclude that we found no evidence that early performance monitoring functions of the pMFC (as reflected by the FRN and FM $\theta$ in the FRN time range) are differentially engaged by face cues that are predictive for upcoming evaluation rules and, therefore, action outcomes. However, such face cues appear to influence later processes of anticipatory control of the pMFC.

\section{Task performance stage}

Analyses of the response-locked EEG data revealed different effect patterns for the time domain and time-frequency domain. Regarding ERN amplitude in the postresponse time window $(0-100 \mathrm{~ms})$, we did not observe the hypothesized amplitude increase when time estimation was going to be assessed by the strict evaluator. However, we observed a significant increase in FM $\theta$ power shortly after the response $(0$ $200 \mathrm{~ms}$ ) in the strict evaluator context. As the ERN has been interpreted as a phase-locked manifestation of theta activity (e.g., Cavanagh et al., 2012), it could be concluded that the observed FM $\theta$ effect rather reflects non-phase-locked proportions of theta that are averaged out when calculating ERPs. Thus, at least for the time-frequency domain we can say that face-induced expectancies about the upcoming evaluation appear to influence early postresponse mechanism of performance monitoring in the pMFC. In particular, although in our task participants could not directly infer from behavior whether it is correct or incorrect, actions assessed by the strict evaluator induced a similar electrocortical responses as have previously been reported for clear behavioral errors (i.e., actions that were wrong in terms of an explicit task rule; e.g. Luu et al., 2004; Mueller, Makeig, Stemmler, Hennig, \& Wacker, 2011; Trujillo \& Allen, 2007). This does not necessarily mean that performance in the strict evaluator context has been rather assessed as false by the system. It is also possible that the process behind this postresponse FM $\theta$ effect has been initialized before the response and does not reflect an error-signal but a genuine "need for control" signal and the implementation thereof (Cavanagh \& Frank, 2014). This could also explain why performance was slightly better under the supervision of the strict evaluator.

Interestingly, neither for ERN amplitude nor for FM $\theta$ power we could detect an influence of performance precision briefly after the response. Hence, the quality of time estimation in our task did not appear to influence performance monitoring functions in the early postresponse time window that has been typically analyzed in the EEG literature on error processing.

However, our rather exploratory analyses of preresponse ERP amplitudes (-160-0 ms) revealed a negative-going and fronto-central deflection for more accurate compared to more imprecise performance. As the time point of the response in our analyses refers to the time when the computer detects the button press, the initiation of the actual motor response must have happened some milliseconds before. This relative negativity for more precise performance should therefore rather be considered as a periresponse deflection. Its scalp topography 
is consistent with a source in the pMFC. It may therefore reflect an increased signaling of need for control by the pMFC which is instantaneously implemented by other, more executive regions leading to benefits in performance (Cavanagh \& Frank, 2014). However, it may also reflect the implementation of control in the pMFC itself (e.g., Aarts, Roelofs, \& Turennout, 2008; Roelofs, van Turennout, \& Coles, 2006; Ruiz, Strübing, Jabusch, \& Altenmüller, 2011). Finally, we observed higher FM $\theta$ power for precise compared to more imprecise performance in a later time window, 400 to $600 \mathrm{~ms}$ following the response. Again, this effect may reflect prolonged signaling of need for control or the implementation thereof (Cavanagh \& Frank, 2014). Certainly, more research is needed to verify the reliability and functional significance of these effects of performance precision in the time domain and time-frequency domain.

Altogether, the observed effects at the performance stage rather indicate that, in our task, monitoring functions of the pMFC were more engaged when performance was more accurate. This is rather inconsistent with classical models of ERN-generation (e.g., Falkenstein et al., 1991; Gehring et al., 1993; Holroyd \& Coles, 2002; Yeung, Botvinick, \& Cohen, 2004), which all predict higher performance monitoring related activity in the pMFC when performance is suboptimal.

It may be noted, however, that in most prior studies on the ERN individuals could directly infer from behavior whether it is correct or incorrect and this was not the case in the time estimation task used here. With regard to our main research topic we can conclude that face induced expectancies influenced early response-locked monitoring mechanism as indexed by FM $\theta$.

\section{Feedback reception stage}

Finally, our findings for the feedback-locked EEG data clearly indicate that face-induced expectancies in our task influence outcome processing by the pMFC. In general, this is in line with several prior studies demonstrating such influence for simpler visual cue stimuli (e.g., Bellebaum \& Daum, 2008; Martin \& Potts, 2011; Mas-Herrero \& Marco-Pallarés, 2014; Pfabigan et al., 2011; Pfabigan et al., 2015; Potts et al., 2006; Walsh \& Anderson, 2011). More specifically, the observed patterns in the feedback-locked FRN and FM $\theta$ power suggest that outcome processing in the pMFC is sensitive to different types of reward prediction errors. First, we observed the typical effects of more negative FRN amplitude and higher FM $\theta$ power in response to negative compared to positive feedbacks. These valence effects are likely to reflect a basal bad-versusgood evaluation, which signals that a current action plan is inadequate and needs to be adjusted (Hajcak, Moser, Holroyd, \& Simons, 2006; Holroyd, Hajcak, \& Larsen, 2006). Second and more important, besides these similar valence effects for the FRN and FM $\theta$, we also observed differential modulations of these two signatures by face-induced expectancies. In detail, our results for the FRN indicate that it is more sensitive to negative RPEs whereas our findings for FM $\theta$ power show that it is rather linked to unsigned RPEs. This pattern does not only add to some recent empirical observations of functional dissociations between feedback-locked FRN and FM $\theta$ (e.g., Hajihosseini \& Holroyd, 2013; Mueller et al., 2014). It also indicates that the pMFC does not homogeneously code for RPEs. As we have recently discussed (Osinsky et al., 2012), the pMFC probably encompasses several functionally distinct populations of neurons, each of which may play a particular role in outcome evaluation. While some of these populations might be more sensitive to signed RPEs (e.g., Matsumoto, Matsumoto, Abe, \& Tanaka, 2007) others may be more sensitive to unsinged RPEs (e.g., Hayden, Heilbronner, Pearson, \& Platt, 2011). It is also possible, that different populations contribute differentially to phase-locked and non-phaselocked portions of the scalp-recorded EEG. Depending on which of these populations is more engaged in a specific task, FRN and/or FM $\theta$ could either be more reflective of positive, negative, or unsigned RPEs. This could also explain the somewhat inconsistent findings in prior research on this topic (see the introduction). Altogether, our feedback-locked EEG results clearly show that early mechanisms of outcome evaluation in the pMFC are sensitive to expectancies which are based on prior experience with a certain evaluator and activated by the sight of this person's face.

\section{Summary and conclusion}

The present study shows that experience-based expectancies about performance evaluation by others influence basal functions of performance monitoring of the $\mathrm{pMFC}$ at various stages of a prototypical assessment situation. First, evaluative expectancies are activated by the sight of the evaluator's face, which acts as a visual cue, predicting the probable outcome of the forthcoming action. When the individual expects a strict evaluation rule, anticipatory processes of performance monitoring appear to be more strongly engaged as indicated by a fronto-central CNV-like amplitude shift. At the following stage of task performance, the expectation of a strict evaluation rule leads to an increase in response-locked FM $\theta$ power, probably reflecting an increased signaling of need for control and/or implementation thereof. Finally, at the stage of feedback reception the face-induced expectations can either be matched or violated, the latter case resulting in RPEs. These RPEs influence early mechanisms of outcome evaluation in the pMFC, as reflected by the feedback-locked FRN and FM $\theta$ power. However, while the FRN rather reflects negative RPEs (i.e., the outcome is worse than expected), FM $\theta$ power is sensitive to unsigned RPEs (i.e., the outcome is other than expected). 
Future studies may further investigate how characteristics like emotional expression, gender, or ethnicity moderate the impact of face cues on performance monitoring. For instance, it would be interesting to see whether such external face attributes interact with more internal, experience-based expectancies like the ones investigated in our study. Moreover, future research may also address the potentially causal role of cue-evoked emotional processes on performance monitoring functions of the pMFC. According to a recent proposal by Inzlicht, Bartholow, and Hirsch (2015), transient increases in negative affect are not only epiphenomenal byproducts of prompts of cognitive control (e.g., errors or conflicts) but do per se alert the organism to an increased need for control. Accordingly, the observed increases in the facelocked CNV amplitude and response-locked FM $\theta$ power following the strict evaluator's face may be a consequence of a transient increase in negative affect.

In general, our study provides further insights into the effects of cue-evoked outcome expectancies on neural functions of performance monitoring. Thereby, the present findings also add to a growing literature on how monitoring functions of the pMFC are influenced by contextual factors. In another recent work, we introduced a threefold taxonomy of contextual factors (global, intermediate, and local) and their relation to the feedback-locked FRN (Osinsky et al., 2014). Accordingly, the face at the beginning of each trial of our time-estimation task can be considered as intermediate contextual information that is based on a continuous integration of prior experiences (e.g., prior encounters with an evaluator). Such intermediate contextual information (together with more global, instructed knowledge) appears to be crucial in determining internal reference systems of action and outcome evaluation in the pMFC, which, in turn, are reflected by the observed electrocortical effects.

\section{References}

Aarts, E., Roelofs, A., \& van Turennout, M. (2008). Anticipatory activity in anterior cingulate cortex can be independent of conflict and error likelihood. Journal of Neuroscience, 28, 4671-4678. doi:10.1523/ JNEUROSCI.4400-07.2008

Bellebaum, C., \& Daum, I. (2008). Learning-related changes in reward expectancy are reflected in the feedback-related negativity. European Journal of Neuroscience, 27(7), 1823-1835. doi:10. 1111/j.1460-9568.2008.06138.x

Brunia, C., van Boxtel, G. J. M., \& Böcker, K. B. (2012). Negative slow waves as indices of anticipation: The Bereitschaftspotential, the contingent negative variation, and the stimulus-preceding negativity. In S. J. Luck \& E. S. Kappenman (Eds.), The Oxford handbook of event-related potential components (pp. 189-207). New York, NY: Oxford University Press.
Capa, R. L., Bouquet, C. A., Dreher, J.-C., \& Dufour, A. (2013). Longlasting effects of performance-contingent unconscious and conscious reward incentives during cued task-switching. Cortex, 49(7), 1943-1954. doi:10.1016/j.cortex.2012.05.018

Carter, C.S, \& van Veen, V. (2007). Anterior cingulate cortex and conflict detection: an update of theory and data. Cognitive Affective and Behavioral Neuroscience, 7, 367-379.

Cavanagh, J. F., Eisenberg, I., Guitart-Masip, M., Huys, Q., \& Frank, M. J. (2013). Frontal theta overrides Pavlovian learning biases. Journal of Neuroscience, 33(19), 8541-8548. doi:10.1523/JNEUROSCI. 5754-12.2013

Cavanagh, J. F., Figueroa, C. M., Cohen, M. X., \& Frank, M. J. (2012a). Frontal theta reflects uncertainty and unexpectedness during exploration and exploitation. Cerebral Cortex, 22, 2575-2586. doi:10. 1093/cercor/bhr332

Cavanagh, J. F., \& Frank, M. J. (2014). Frontal theta as a mechanism for cognitive control. Trends in Cognitive Sciences, 18, 414-421. doi: 10.1016/j.tics.2014.04.012

Cavanagh, J. F., Frank, M. J., Klein, T. J., \& Allen, J. J. B. (2010). Frontal theta links prediction errors to behavioral adaptation in reinforcement learning. NeuroImage, 49, 3198-3209. doi:10.1016/j. neuroimage.2009.11.080

Cavanagh, J. F., Zambrano-Vazquez, L., \& Allen, J. J. B. (2012b). Theta lingua franca: A common mid-frontal substrate for action monitoring processes. Psychophysiology, 49, 220-238. doi:10.1111/j.14698986.2011.01293.x

Chase, H. W., Swainson, R., Durham, L., Benham, L., \& Cools, R. (2011). Feedback-related negativity codes prediction error but not behavioral adjustment during probabilistic reversal learning. Journal of Cognitive Neuroscience, 23(4), 936-946. doi:10.1162/ jocn.2010.21456

Cohen, M. X., Elger, C. E., \& Ranganath, C. (2007). Reward expectation modulates feedback-related negativity and EEG spectra. NeuroImage, 35, 968-978. doi:10.1016/j.neuroimage.2006.11.056

Debener, S., Ullsperger, M., Siegel, M., Fiehler, K., von Cramon, D. Y., \& Engel, A. K. (2005). Trial-by-trial coupling of concurrent electroencephalogram and functional magnetic resonance imaging identifies the dynamics of performance monitoring. Journal of Neuroscience, 25(50), 11730-11737. doi:10.1523/JNEUROSCI.3286-05.2005

Dunning, J. P., \& Hajcak, G. (2007). Error-related negativities elicited by monetary loss and cues that predict loss. Neuroreport, 18(17), 18751878. doi:10.1097/WNR.0b013e3282f0d50b

Falkenstein, M., Hohnsbein, J., Hoormann, J., \& Blanke, L. (1991). Effects of crossmodal divided attention on late ERP components: II. Error processing in choice reaction tasks. Electroencephalography and Clinical Neurophysiology, 78, 447455. doi:10.1016/0013-4694(91)90062-9

Ferdinand, N. K., Mecklinger, A., Kray, J., \& Gehring, W. J. (2012). The processing of unexpected positive response outcomes in the mediofrontal cortex. Journal of Neuroscience, 32, 12087-12092. doi:10.1523/JNEUROSCI.1410-12.2012

Gehring, W. J., Goss, B., Coles, M. G., Meyer, D. E., \& Donchin, E. (1993). A neural system for error detection and compensation. Psychological Science, 4(6), 385-390. doi:10.1111/j.1467-9280. 1993.tb00586.x

Gehring, W. J., \& Willoughby, A. R. (2002). The medial frontal cortex and the rapid processing of monetary gains and losses. Science, 295 , 2279-2282. doi: $10.1126 /$ science 1066893

Hajcak, G., Moser, J. S., Holroyd, C. B., \& Simons, R. F. (2006). The feedback-related negativity reflects the binary evaluation of good versus bad outcomes. Biological Psychology, 71(2), 148-154. doi: 10.1016/j.biopsycho.2005.04.001

Hajihosseini, A., \& Holroyd, C. B. (2013). Frontal midline theta and N200 amplitude reflect complementary information about expectancy and outcome evaluation. Psychophysiology, 50, 550-562. doi:10. 1111/psyp.12040 
Hauser, T., Iannaccone, R., Stämpfli, P., Drechsler, R., Brandeis, D., Walitza, S., \& Brem, S. (2014). The feedback-related negativity (FRN) revisited: New insights into the localization, meaning and network organization. NeuroImage, 84, 159-168. doi:10.1016/j. neuroimage.2013.08.028

Hayden, B. Y., Heilbronner, S. R., Pearson, J. M., \& Platt, M. L. (2011). Surprise signals in anterior cingulate cortex: Neuronal encoding of unsigned reward prediction errors driving adjustment in behavior. Journal of Neuroscience, 31(11), 4178-4187. doi:10.1523/ JNEUROSCI.4652-10.2011

Hewig, J., Trippe, R., Hecht, H., Coles, M. G. H., Holroyd, C. B., \& Miltner, W. H. R. (2007). Decision-making in blackjack: An electrophysiological analysis. Cerebral Cortex, 17(4), 865-877. doi:10. 1093/cercor/bhk040

Holroyd, C. B., \& Coles, M. G. H. (2002). The neural basis of human error processing: Reinforcement learning, dopamine, and the errorrelated negativity. Psychological Review, 109(4), 679-709. doi:10. 1037/0033-295X.109.4.679

Holroyd, C. B., Hajcak, G., \& Larsen, J. T. (2006). The good, the bad and the neutral: Electrophysiological responses to feedback stimuli. Brain Research, 1105(1), 93-101. doi:10.1016/j.brainres.2005.12. 015

Holroyd, C. B., Krigolson, O. E., \& Lee, S. (2011). Reward positivity elicited by predictive cues. NeuroReport, 22, 249-252. doi:10.1097/ WNR.0b013e328345441d

Inzlicht, M., Bartholow, B.M., \& Hirsh, J.B. (2015). Emotional foundations of cognitive control. Trends in Cognitive Sciences, 19, 126132. doi:10.1016/j.tics.2015.01.004

Langner, O., Dotsch, R., Bijlstra, G., Wigboldus, D. H. J., Hawk, S. T., \& van Knippenberg, A. (2010). Presentation and validation of the Radboud Faces Database. Cognition \& Emotion, 24(8), 13771388. doi:10.1080/02699930903485076

Liao, Y., Gramann, K., Feng, W., Deák, G. O., \& Li, H. (2011). This ought to be good: Brain activity accompanying positive and negative expectations and outcomes. Psychophysiology, 48, 1412-1419. doi:10.1111/j.1469-8986.2011.01205.x

Luu, P., Tucker, D. M., \& Makeig, S. (2004). Frontal midline theta and the error-related negativity: Neurophysiological mechanisms of action regulation. Clinical Neurophysiology, 115(8), 1821-1835. doi:10. 1016/j.clinph.2004.03.031

Marco-Pallarés, J., Cucurell, D., Cunillera, T., García, R., Andrés-Pueyo, A., Münte, T. F., \& Rodríguez-Fornells, A. (2008). Human oscillatory activity associated to reward processing in a gambling task. Neuropsychologia, 46(1), 241-248. doi:10.1016/j. neuropsychologia.2007.07.016

Martin, L. E., \& Potts, G. F. (2011). Medial frontal event-related potentials and reward prediction: Do responses matter? Brain and Cognition, 77(1), 128-134. doi:10.1016/j.bandc.2011.04.001

Mas-Herrero, E., \& Marco-Pallarés, J. (2014). Frontal theta oscillatory activity is a common mechanism for the computation of unexpected outcomes and learning rate. Journal of Cognitive Neuroscience, 26(3), 447-458. doi:10.1162/jocn_a_00516

Matsumoto, M., Matsumoto, K., Abe, H., \& Tanaka, K. (2007). Medial prefrontal cell activity signaling prediction errors of action values. Nature Neuroscience, 10(5), 647-656. doi:10.1038/nn1890

Miltner, W. H., Braun, C. H., \& Coles, M. G. (1997). Event-related brain potentials following incorrect feedback in a time-estimation task: Evidence for a "generic" neural system for error detection. Journal of Cognitive Neuroscience, 9(6), 788-798. doi:10.1162/ jocn.1997.9.6.788

Mueller, E. M., Burgdorf, C., Chavanon, M.-L., Schweiger, D., Wacker, J., \& Stemmler, G. (2014). Dopamine modulates frontomedial failure processing of agentic introverts versus extraverts in incentive contexts. Cognitive, Affective, \& Behavioral Neuroscience, 14, 756-768. doi:10.3758/s13415-013-0228-9
Mueller, E. M., Makeig, S., Stemmler, G., Hennig, J., \& Wacker, J. (2011). Dopamine effects on human error processing depend on Catechol-O-methyltransferase VAL158MET genotype. Journal of Neuroscience, 31, 15818-15825. doi:10.1523/JNEUROSCI.210311.2011

Mussel, P., Reiter, A. M., Osinsky, R., \& Hewig, J. (2015). State- and trait-greed, its impact on risky decision-making and underlying neural mechanisms. Social Neuroscience, 10(2), 126-134. doi:10.1080/ 17470919.2014.965340

Nieuwenhuis, S., Schweizer, T. S., Mars, R. B., Botvinick, M. M., \& Hajcak, G. (2007). Error-likelihood prediction in the medial frontal cortex: A critical evaluation. Cerebral Cortex, 17, 1570-1581. doi: 10.1093/cercor/bhl068

Oliveira, F. T. P., Hickey, C., \& McDonald, J. J. (2014). Proactive and reactive processes in the medial frontal cortex: An electrophysiological study. PLOS ONE, 9(1), e84351. doi:10.1371/journal.pone. 0084351

Oliveira, F. T. P., McDonald, J. J., \& Goodman, D. (2007). Performance monitoring in the anterior cingulate is not all error related: Expectancy deviation and the representation of action-outcome associations. Journal of Cognitive Neuroscience, 19(12), 1994-2004. doi:10.1162/jocn.2007.19.12.1994

Osinsky, R., Mussel, P., Ohrlein, L., \& Hewig, J. (2014a). A neural signature of the creation of social evaluation. Social Cognitive and Affective Neuroscience, 9(6), 731-736. doi:10.1093/scan/nst051

Osinsky, R., Mussel, P., \& Hewig, J. (2012). Feedback-related potentials are sensitive to sequential order of decision outcomes in a gambling task. Psychophysiology, 49, 1579-1589. doi:10.1111/j.1469-8986. 2012.01473.x

Osinsky, R., Walter, H., \& Hewig, J. (2014b). What is and what could have been: An ERP study on counterfactual comparisons. Psychophysiology, 51, 773-781. doi:10.1111/psyp.12221

Pailing, P. E., \& Segalowitz, S. J. (2004). The effects of uncertainty in error monitoring on associated ERPs. Brain and Cognition, 56(2), 215-233. doi:10.1016/j.bandc.2004.06.005

Pfabigan, D. M., Alexopoulos, J., Bauer, H., \& Sailer, U. (2011). Manipulation of feedback expectancy and valence induces negative and positive reward prediction error signals manifest in event-related brain potentials. Psychophysiology, 48(5), 656-664. doi:10.1111/j. 1469-8986.2010.01136.x

Pfabigan, D. M., Seidel, E.-M., Paul, K., Grahl, A., Sailer, U., Lanzenberger, R., \& Lamm, C. (2015). Context-sensitivity of the feedback-related negativity for zero-value feedback outcomes. Biological Psychology, 104, 184-192. doi:10.1016/j.biopsycho. 2014.12.007

Potts, G. F., Martin, L. E., Burton, P., \& Montague, P. R. (2006). When things are better or worse than expected: The medial frontal cortex and the allocation of processing resources. Journal of Cognitive Neuroscience, 18(7), 1112-1119. doi:10.1162/jocn.2006.18.7.1112

Roelofs, A., van Turennout, M., \& Coles, M. G. H. (2006). Anterior cingulate cortex activity can be independent of response conflict in Stroop-like tasks. Proceedings of the National Academy of Sciences, 103, 13884-13889. doi:10.1073/pnas.060626510

Ruiz, M. H., Strübing, F., Jabusch, H.-C., \& Altenmüller, E. (2011). EEG oscillatory patterns are associated with error prediction during music performance and are altered in musician's dystonia. NeuroImage, 55, 1791-1803. doi:10.1016/j.neuroimage.2010.12.050

Sambrook, T. D., \& Goslin, J. (2015). A neural reward prediction error revealed by a meta-analysis of ERPs using great grand averages. Psychological Bulletin, 141(1), 213-235. doi:10. 1037/bul0000006

Scheffers, M. K., \& Coles, M. (2000). Performance monitoring in a confusing world: Error-related brain activity, judgments of response accuracy, and types of errors. Journal of Experimental Psychology: Human Perception and Performance, 26, 141-151. doi:10.1037/0096-1523.26.1.141 
Schevernels, H., Krebs, R. M., Santens, P., Woldorff, M. G., \& Boehler, C. N. (2014). Task preparation processes related to reward prediction precede those related to task-difficulty expectation. NeuroImage, 84, 639-647. doi:10.1016/j.neuroimage.2013.09.039

Shackman, A. J., Salomons, T. V., Slagter, H. A., Fox, A. S., Winter, J. J., \& Davidson, R. J. (2011). The integration of negative affect, pain and cognitive control in the cingulate cortex. Nature Reviews Neuroscience, 12(3), 154-167. doi:10.1038/nrn2994

Silvetti, M., Nuñez Castellar, E., Roger, C., \& Verguts, T. (2014). Reward expectation and prediction error in human medial frontal cortex: An EEG study. NeuroImage, 84, 376-382. doi:10.1016/j.neuroimage. 2013.08.058

Tallon-Baudry, C., Bertrand, O., Peronnet, F., \& Pernier, J. (1998). Induced gamma-band activity during the delay of a visual short-term memory task in humans. Journal of Neuroscience, 18(11), 4244-4254.

Trujillo, L. T., \& Allen, J. J. (2007). Theta EEG dynamics of the errorrelated negativity. Clinical Neurophysiology, 118(3), 645-668. doi: 10.1016/j.clinph.2006.11.009

Ullsperger, M., Danielmeier, C., \& Jocham, G. (2014). Neurophysiology of performance monitoring and adaptive behavior. Physiological Reviews, 94(1), 35-79. doi:10.1152/physrev.00041.2012

van den Berg, B., Krebs, R. M., Lorist, M. M., \& Woldorff, M. G. (2014). Utilization of reward-prospect enhances preparatory attention and reduces stimulus conflict. Cognitive, Affective, \& Behavioral Neuroscience, 14(2), 561-577. doi:10.3758/s13415-014-0281-z

Walsh, M. M., \& Anderson, J. R. (2011). Learning from delayed feedback: Neural responses in temporal credit assignment. Cognitive,
Affective, \& Behavioral Neuroscience, 11, 131-143. doi:10.3758/ s13415-011-0027-0

Walsh, M. M., \& Anderson, J. R. (2012). Learning from experience: Event-related potential correlates of reward processing, neural adaptation, and behavioral choice. Neuroscience and Biobehavioral Reviews, 36, 1870-1884. doi:10.1016/j. neubiorev.2012.05.008

Walter, W. G., Cooper, R., Aldridge, V. J., McCallum, W. C., \& Winter, A. L. (1964). Contingent negative variation: An electric sign of sensorimotor association and expectancy in the human brain. Nature, 203, 380-384.

Wu, Y., \& Zhou, X. (2009). The P300 and reward valence, magnitude, and expectancy in outcome evaluation. Brain Research, 1286, 114 122. doi:10.1016/j.brainres.2009.06.032

Xu, Q., Shen, Q., Chen, P., Ma, Q., Sun, D., \& Pan, Y. (2011). How an uncertain cue modulates subsequent monetary outcome evaluation: An ERP study. Neuroscience Letters, 505(2), 200-204. doi:10.1016/ j.neulet.2011.10.024

Yeung, N., Botvinick, M. M., \& Cohen, J. D. (2004). The neural basis of error detection: Conflict monitoring and the error-related negativity. Psychological Review, 111(4), 931-959. doi:10.1037/0033-295X. 111.4.931

Yu, R., \& Zhou, X. (2009). To bet or not to bet? The error negativity or error-related negativity associated with risk-taking choices. Journal of Cognitive Neuroscience, 21, 684-696. doi:10.1162/jocn.2009. 21034 